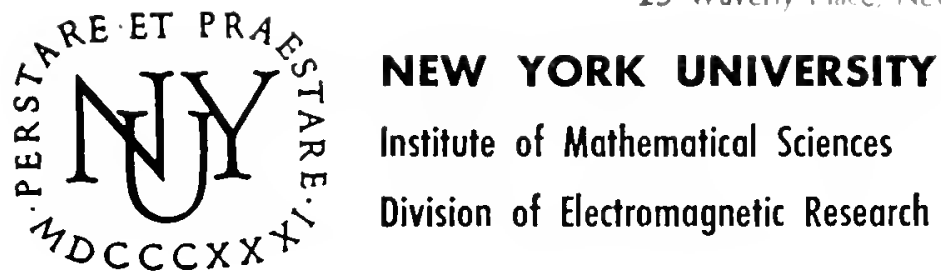

RESEARCH REPORT No. HSN-3

\title{
The Closure Problem of Turbulence Theory
}

\author{
ROBERT H. KRAICHNAN
}

Contract No. N(onr)-285(33)

JANUARY, 1961 
NE'W YORK UNTVERSITY

Institute of Mathematical Sciences

Division of Electromagnetic Research

The Closure Problem of Turbulence Theory ${ }^{*}$

Robert H. KraIchnan

Research Report No. HSN-3

This work was supported by the Office of Naval Research under Contract No. Nonr-285(33).
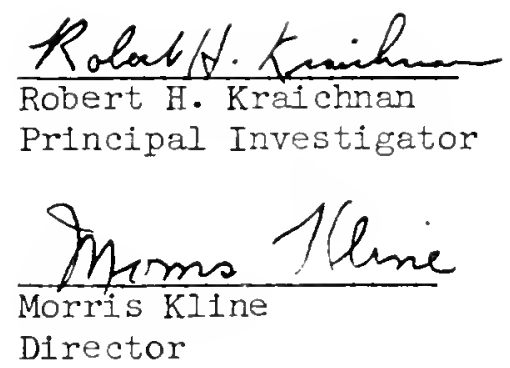

$$
\text { January, } 1961
$$

*

Thys paper was presented at the Symposium on Hydrodynamic Instability sponsored by the American Mathematical Society in New York City, April 1960. The Proceedings of the Symposlum will be published by the American Mathematical Soclety (Volume 13, Garrett BIrkhoff editor, Proceedings of Symposia in Applied Mathematics). 


\section{ABSTRACT}

An examination is made of the physical bases for the description of turbulence by probability distributions, and for the closure problem which arises from this description. Then a discussion is given of the analytical approaches to the closure problem which have been proposed by Deissler, Hopf, Kralchnan, Proudman and Reid, and Tatsumi. Some mathematical inter-relations of these schemes are cited. Certaln consequences of the several approaches are illustrated by applying them to an idealized turbulent convection problem for which they all yleld explicit solutions.

\section{CONTENTS}

\section{Introduction}

2. The Physical Nature of Turbulence

3. Mathematical Formulation

4. The Closure Problem

5. Schemes for Approximate Closure 14

5.1. Isotropic turbulence

5.2. Expansion in powers of Reynolds number

5.3. Expansion in cumulants 19

5.4. Approximation by stochastic models

6. An Application of the Closure Approximations 27

6.1. Convection by a random velocity field 27

6.2 Iteration expansion 31

6.3. Cumulant-discard approximations 34

6.4. Stochastic model approximations 39

6.5. Formal relations among the approximations 41

7. Conclusion 43

References $\quad 46$ 



\section{INTRODUCTION}

Turbulent flows which occur in nature, or the laboratory, can appear Inflnitely complex to the eye. This is faithfully reflected in turbulence theory. A central feature of the theory is an inflnite set of dynamical equations which couple together all the moments of a statistically distributed velocity field. The closure problem assoclated with these equations may be posed as follows: How are they to be replaced by a fintte set that yields the limited statistical information of actual interest? This question must be answered, explicltly or implicitly, in order to solve most problems in turbulence theory. Consequently, the closure problem has received a great deal of attention since it flrst confronted Reynolds [23] and Taylor $[29]$.

The present paper opens with an examination of the physical bases for the mathematical description of turbulence by probability distributions, and for the closure problem which arises from this descr: stion. After discussing these matters, we shall take up analytical approaches to the closure problem which have been proposed by Deissler $[7,8]$, Hopf $[10,11]$, Kraichnan $[15,16]$, Proudman and Reid $[21,22]$, and Tatsum $[27,28]$. We shall describe some mathematical inter-relations and then 1llustrate certain consequences of the several approaches by applying them to an idealized turbulent convection problem for which they all yleld explicit solutions.

\section{THE PHYSICAL NATURE OF IURBULENCE}

Fluid flows which are called turbulent by fluid dynamicists typlcally have two related characteristics: a complicated variation of velocity with position and time, and an instability to small disturbances. In experiments, 
many kinds of small disturbances are present and uncontrollable. Thus, the instability makes it impossible to predict the velocity at any given time and place from a knowledge of the gross initial and boundary conditions.

Let us consider the classic example of water which flows from a reservolr through a long circular pipe into a second reservoir at lower pressure. I Let this experiment be repeated many times in as closely identical fashion as possible. If the flow is slow enough, it 1 s fully laminar: The velocity varies smoothly and monotonically from wall to axis of the pipe, and it is very nearly reproducible from one trial (realization) of the experiment to another. ${ }^{2}$ If the flow is fast enough, however, the velocity in a typical realization displays complicated smallscale variations in space and time, and these variations are found to change unpredictably from one realization to the next. At a representative point in the pipe, the measured velocity in a typical realization will differ by several percent from the mean velocity at that point obtained by averaging over an ensemble of very many realizations. Moreover, the velocity fluctuations remain at this level no matter how hard we try to keep the experimental conditions identical to all realizations. We shall now define the turbulent velocity field as the difference between the actual velocity field in a given realization and the ensemble-mean velocity field. Its essential characteristic is unpredictability under

${ }^{1}$ For a detailed description of turbulent pipe flow, see Laufer [I7] and Townsend $[32]$.

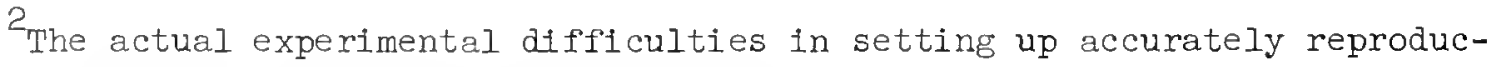
ible laminar flows can be very severe. 
attainable control of experimental conditions.

As we have stated, the reason for the appearance of the turbulent velocity fleld appears to be instability to small disturbances. These include fluctuations in temperature and density of the fluid, velocity fluctuations in the reservoir (where supposedly the fluid is at rest), and coupling of the flow to vibrations of the walls of the pipe. The dimensionless parameter which principally determines whether the flow is stable or not is the Reynolds number DU/v, where D is the pipe diameter, $U$ the mean velocity on axis, and $v$ the kinematic viscosity. For low enough Reynolds number, the velocity perturbations in the pipe, induced by the sources just cited, are damped out by viscosity. For high enough Reynolds number, the viscous friction is overbalanced by the shearing action of the existing flow upon the perturbation velocity [18]. Thts action causes the perturbation velocity to draw energy from the existing flow and grow rapidly into the macroscopic fluctuations which characterize the turbulent state. The process is demonstrated daily for those of us who stir milk into our coffee.

The pipe flow experiment is representative of actual turbulent shear flows in that the turbulent velocity at any point is typically only a few percent of the mean velocity, no matter how high the Reynolds number is. Its presence, however, is associated with a profound change in the character of the mean flow. In the fully laminar state, the mean velocity profile across the pipe is parabolic. In the turbulent state at high Reynolds numbers, the mean velocity is almost constant over most of the pipe crosssection and falls very rapidly to zero in a narrow boundary layer at the wall. The flow resistance is very high compared to that in the laminar 
state, and, except in the immediate vicinity of the wall, the radial transport of momentum is dominated by the turbulent velocity rather than by viscosity.

The unpredictability and complicated form of the turbulent velocity field lead one to describe it by probability distributions over a large ensemble of realizations. Even if the exact, detailed structure of a single realization could be measured, it would never be reproduced in any repetition of the experimert.

A paradoxical feature of turbulence, but one which is very fortunate for the theorist, is that extreme sensitivity of individual realizations to small perturbations is accompanied by an insensitivity of the probability alstribution to the nature and probability distribution of the perturbations. Suppose that we place in the entrance to the pipe a grid of wires which substantially disturbs the flow and thus dominates the perturbations which lead to turbulent breakdown. In a region downstream of the grid, the probability distribution of the turbulent velocity will depend on the parameters of the grid. Further downstream, however, the probability distribution will be almost independent of the nature of the grid, and of its presence, provided the Reynolds number is high enough. We shall call the region of sensitivity to the nature of the triggering disturbances the transition region, and the region of insensitivity that of fully-developed turbulence. In the latter region, the probability distribution of the turbulent velocity is, in adaltion, highly insensitive to small disturbances of a local nature. The insensitivity is only statistical, however. In any realization, the detalled velocity field is highly unstable to small disturbances. The qualification should also be raised 
that the observed statistical insensitivity might be less striking if more detalled and accurate statistical measurements could be made.

\section{MATHEMATICAL FORMULATION}

Let us write the total velocity fleld in a realization as

$$
v_{i}(\underset{m}{x}, t)=\vec{v}_{i}(\underset{m}{x}, t)+u_{i}(\underset{m}{x}, t),
$$

where $\vec{v}_{1}(x, t)$ is the ensemble-average of $v_{i}(\underset{m}{x}, t)$ and, by our previous definition, $u_{1}(\underset{m}{x}, t)$ is then the turbulent velocity. The usual statistical description of $u_{i}(x, t)$ is by the infinite set of moments

$$
\begin{aligned}
& U_{1 j}\left(\underset{m}{x}, t ; x_{m}^{\prime}, t^{\prime}\right)=\left\langle u_{1}(\underset{m}{x}, t) u_{j}\left(x_{m}^{\prime}, t^{\prime}\right)\right\rangle, \\
& U_{i j m}\left(x_{m}^{x}, t ; x_{m}^{\prime}, t^{\prime} ; x_{m}^{\prime \prime}, t^{\prime \prime}\right)=\left\langle u_{i}(x, t) u_{j}\left(x_{m}^{\prime}, t^{\prime}\right) u_{m}\left(x_{m}^{\prime \prime}, t^{\prime \prime}\right)\right\rangle,
\end{aligned}
$$

where 〈〉 denotes ensemble average. A more compact description, but one which so far has not been much used, is by the distribution functions $P_{1}(\underset{m}{u}, x, t), P_{2}\left(\underset{m}{u, x}, t ; u_{m}^{\prime}, x_{m}^{\prime}, t^{\prime}\right)$, etc. deflned as follows: $P_{1}\left({\underset{m}{m}}_{m}, x_{m}\right) d^{3} u$ is the probability that $\underline{\mathrm{u}}(\mathrm{x}, \mathrm{t})$ lies in the volume $\mathrm{d}^{3} \mathrm{u}$ about the point $\mathrm{u}_{\mathrm{m}}$ in velocity space, $P_{2}\left(\underset{m}{u}, x, t ; \underset{m}{u^{\prime}}, x_{m}^{\prime}, t^{\prime}\right) d^{3} u d^{3} u^{\prime}$ is the joint probability that $u(x, t)$ lies within the volume $d^{3} u$ at $\underset{\sim}{u}$ and ${\underset{w}{u}}_{u}\left(x^{\prime}, t^{\prime}\right)$ lies within the volume $d^{3} u^{\prime}$ at $\mathrm{u}^{\prime}$, etc. Each $\mathrm{P}_{\mathrm{n}}$ is equivalent to an infinite subset of the moments (3.2). (Experimental measurements have been made of both moments and distribution functions.)

We may now state the theoret1cal problem of turbulence as follows: We are given the Initial mean flow $\bar{v}_{1}\left(\underset{w}{x}, t_{0}\right)$, together with the complete set of moments which specify the distribution of the initial turbulent 
velocity field pru $\left(\underset{m}{x}, t_{0}\right)$ ( If the latter is nonzero). We are also given a complete statistical description, in terms of moments, of whatever external forces or perturbations may be acting during the course of the flow. We wish to find $\bar{v}_{i}(\underset{m}{x}, t)$, together with the complete set of moments (3.2) at all times after $t_{0}$.

Let us assume that the flutd is incompressible, of unit density, and (apart from perturbation effects) obeys the Navier-Stokes equation

(3.3) $\quad\left(\frac{\partial}{\partial t}-v \nabla\right) v_{i}=-v_{m} \frac{\partial v_{i}}{\partial x_{m}}-\frac{\partial p}{\partial x_{i}}$,

where $v$ is a constant kinematic viscosity and $p$ is pressure. The pressure term in (3.3) may be expressed as a end-degree polynomial functional of the velocity fleld by use of the boundary conditions and the divergence condition

(3.4) $\quad \partial v_{1} / \partial x_{1}=0$

This reduction is simplest when the flutd is infinite and $p$ vanishes at Infinity. Then

(3.5) $\quad p(\underset{m}{x}, t)=\frac{1}{4 \pi} \int \mid \underset{m-x_{m}^{\prime}}{\mid-1} \frac{\partial v_{1}\left(x^{\prime}, t\right)}{\partial x_{m}^{\prime}} \frac{\partial v_{m}\left(x_{m}^{\prime}, t\right)}{\partial x_{1}^{\prime}} d^{3} x^{\prime}$,

and (3.3) may be written in the form

(3.6) $\left(\frac{\partial}{\partial t}-v \nabla^{2}\right) v_{i}=-\frac{1}{2} P_{i m n}(\nabla)\left[v_{m} v_{n}\right]$,

where 
$P_{1 m n}(\nabla)=\left(\delta_{i m}-\nabla^{-2} \frac{\partial^{2}}{\partial x_{i} \partial x_{m}}\right) \frac{\partial}{\partial x_{n}}+\left(\delta_{i n}-\nabla^{-2} \frac{\partial^{2}}{\partial x_{1} \partial x_{n}}\right) \frac{\partial}{\partial x_{m}}$

and $\nabla^{-2} f(x)=-(4 \pi)^{-1} \int\left|\underset{\sim}{x-x_{m}}\right|^{-1} f\left(\underset{\sim}{x^{\prime}}\right) d^{3} x^{\prime}$ for any $f$.

We have so far not represented the small disturbances which play so important a role in turbulence. In order to include their effects accurately, we would have to examine in detail the physical mechanisms respons1ble. Instead, we shall be guided by the empirical finding that, In many flows, there exists a fully-turbulent state which is insensitive to the precise nature of the disturbances. The simplest assumption is to lump the effects of all disturbances into distortions of the Initial turbulent velocity field and then assume that (3.3), without additional terms and without perturbations in the boundary conditions, holds exactly throughout the course of the flow. This implies that disturbances durlng the course of the flow merely nudge the system from one solution of (3.3) to a solution corresponding to another initial condition. We shall adopt this specialization of the turbulence problem. We must recognize, however, that it cannot be expected to yield an accurate description of the transition stage of turbulence. Moreover, 1 ts adequacy in representing any turbulent state is only a conjecture which must be tested by 1 ts consequences.

In principle, we may now solve the turbulence problem as follows. We assume an 1 nit1al mean fleld $\underset{\sim}{\vec{v}}\left(\underset{m}{x}, t_{0}\right)$ and 1 nitial values for the set of moments $(3.2)$. Then we construct an inftial ensemble of fields $\underset{\sim}{v}\left(x_{\sim}, t_{0}\right)+$ 
$\underset{\sim}{u}\left(\underset{m}{x}, t_{0}\right)$ which yield these moments. We solve (3.3) for each of these Inftial conditions (assuming solutions exist for all $t$ ) and, from the solutions, construct $\bar{v}(x, t)$ and those moments (3.2) which are of interest.

This procedure is not a practical one. Few solutions of (3.3) are known even for simple initial conditions. To carry out the above program accurately for a large set of complicated inftial conditions would involve, at present, a prohibitively large numerical computation effort. Even $1 f$ this were done, there would remain the task of abstracting from the mass of calculations an 1nsight into the essential dynamical nature of the turbulence. We are led, instead, to seek a method of solution which takes us directly from the initial set of moments to the values, at later times, of the moments of interest. To do this, we construct, from (3.2), equations of motion for the moments themselves. Therein lies the closure problem which is to be our principal concern.

\section{THE CLOSURE PROBLEM}

Let us now specialize to an infinite fluid for which (3.6) is valid. This w1ll simplify the mathematics, and we may hope that the essential nature of the results wIll be unchanged in the more general case. By averaging (3.6) over the ensemble, we obtain

$$
\begin{gathered}
(4.1) \quad\left(\frac{\partial}{\partial t}-v \nabla^{2}\right) \bar{v}_{1}(\underset{m}{x}, t)+\frac{1}{2} P_{i m n}(\nabla)\left[\bar{v}_{m}(\underset{m}{x}, t) \bar{v}_{n}(\underset{m}{x}, t)\right] \\
=-\frac{1}{2} P_{i m n}(\nabla) U_{m n}(\underset{m}{x}, t ; \underset{m}{x}, t) .
\end{gathered}
$$


Subtracting (4.1) from $(3.6)$, we obtiln

$(4.2)$

$$
\begin{aligned}
\left(\frac{\partial}{\partial t}-v \nabla^{2}\right) u_{i}(x, t)+P_{i m n}(\nabla)\left[\bar{v}_{m}(x, t) u_{n}(x, t)\right] \\
=-\frac{1}{2} P_{i m n}(\nabla)\left[u_{m}(x, t) u_{n}(x, t)-u_{m n}(x, t ; x, t)\right],
\end{aligned}
$$

where we note $P_{1 m n}(\nabla)=P_{i n m}(\nabla)$. Now, by multiplying $(4 \cdot 2)$ with $u_{j}\left(x^{\prime}, t\right)$, $u_{j}\left(x^{\prime}, t\right) u_{r}\left(x_{\sim}^{\prime \prime}, t^{\prime \prime}\right)$, etc. and averaging, we obtain the infinite set of equat1ons

$$
\begin{gathered}
\left(\frac{\partial}{\partial t}-v \nabla_{x}^{2}\right) U_{i j}\left(x, t ; x_{m}^{\prime}, t^{\prime}\right)+P_{1 m n}\left(\nabla_{x}\right)\left[\bar{v}_{m}(x, t) U_{n j}\left(x_{m}, t ; x_{m}^{\prime}, t^{\prime}\right)\right] \\
=-\frac{1}{2} P_{i m n}\left(\nabla_{x}\right)\left[U_{m n j}\left(x, t ; x_{m}^{x}, t ; x_{m}^{\prime}, t^{\prime}\right)\right]
\end{gathered}
$$

$(4.3)$

$$
\begin{aligned}
& \left(\frac{\partial}{\partial t}-v \nabla_{x}^{2}\right) U_{1 j r}\left(\underset{\sim}{x}, t ; \underset{\sim}{x^{\prime}}, t^{\prime} ; x^{\prime \prime}, t^{\prime \prime}\right) \\
& +P_{i m n}\left(\nabla_{x}\right)\left[\vec{v}_{m}(\underset{\sim}{x}, t) U_{n j r}\left(\underset{\sim}{x}, t ; x^{\prime}, t^{\prime} ; x_{\sim}^{\prime \prime}, t^{\prime \prime}\right)\right] \\
& =-\frac{1}{2} P_{i m n}\left(\nabla_{x}\right)\left[U_{m n j r}\left(\underset{\sim}{x}, t ; \underset{\sim}{x}, t ; x_{\sim}^{\prime}, t^{\prime} ; x_{\sim}^{\prime \prime}, t^{\prime \prime}\right)\right. \\
& \left.-U_{m n}(x, t ; \underset{\sim}{x}, t) U_{j r}\left(x_{\sim}^{\prime}, t^{\prime} ; x_{m}^{\prime \prime}, t^{\prime \prime}\right)\right],
\end{aligned}
$$

These equations are formidable in appearance (and not deceptively so!). To show their essent1al structure, we shall rewhte (4.1) and (4.3) 
in the schematic form

$$
\left(4.1^{\prime}\right) \quad\left(\frac{\partial}{\partial t}-v \nabla^{2}\right) \bar{v}+\frac{1}{2} P(\nabla)[\bar{v} \bar{v}]=-\frac{1}{2} U_{2}
$$

and

$\left(4.3^{\prime}\right)$

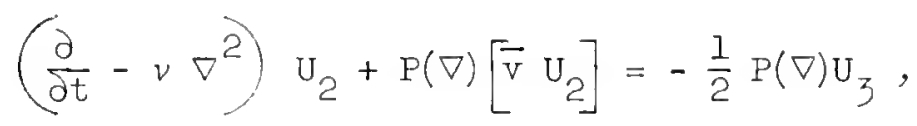

$$
\left(\frac{\partial}{\partial t}-v \nabla^{2}\right) U_{3}+P(\nabla)\left[\bar{v} U_{3}\right]=-\frac{1}{2} P(\nabla)\left[U_{4}-U_{2} U_{2}\right] \text {, }
$$

where $U_{2}$ denotes a second-order moment and so forth. We see that (4.1) and (4.3) are a coupled set of equations that link moments of all orders. In general, the equation for the nth-order moments involves linearly the $(n+1)$ th-order moments. If we consider the integration of these equations forward in time, 1 is clear that the value of any moment at later times is a functional of the initial values of the full infinite set of moments.

The coupling of moments of all orders arises from the right side of $(4.2)$ and is a direct consequence of the nonlinearity of the Navier-Stokes equation. The nonlinearity, in turn, is the mathematical embodiment of the self-stirring of the velocity field. We should not be surprised that the moments are coupled. In any realization the velocity at a given time and place is determined by the entire initial velocity field. It is plausible, then, for the mean velocity at that place to depend on the full Initial probability distribution. 
Our previous discussion suggests, however, that the full dependence of given moments on the stmucture of the initial distribution is far more information then we wat. Indeed, the 1dealization of the turbulence problem as one with stochastic inftial conditions and fully determinate equations of motion was based on the hope that, w1thin limits, the evolution of the turbulence was substantially independent of the detalls of the initial alstribution.

Let us therefore specialize our problem to the case where the inftial distribution is of the simplest possible form: a multivariate normal distribution, which is completely specified by $\bar{v}_{1}\left(x, t_{0}\right)$ and $\mathrm{U}_{1, j}\left(\mathrm{x}_{\sim}, \mathrm{t}_{0} ; \mathrm{x}_{\sim}^{\prime}, \mathrm{t}_{0}\right)$. Eventually we would like to be able to solve the statistical problem for a variety of types of initial distribution, so as to see just what sensitivity to this choice the solutions actually display. The multivariate normal distribution seems a logical starting point because it is simplest and because, in any event, we do not really know what distribution best represents the physical perturbations which occur in experiments.

At this point we come to a fundamental fact which is distressing. Given a multivariate normal initial distribution, it seems clear that any given moment at later times can be expressed as a functional of $\bar{v}_{i}\left(x_{m}, t_{0}\right)$ and $\mathrm{U}_{1 j}\left(\mathrm{x}_{\sim}, t_{0} ; \mathrm{x}_{\sim}, t_{0}\right)$. If, however, we attempt to find the explicit form of, say, $\bar{v}_{i}(\underset{w}{x}, t)$ by integration of the moment equations (4.1) and (4.3) In time, we obtain an infinitely complicated functional of $\bar{v}_{1}\left(\underset{\mu}{x}, t_{0}\right)$ and $U_{i j}\left(x_{m}, t_{0} ; x^{\prime}, t_{0}\right)$. For example, if we do the integration in the most straightforward way, by expansion in a Taylor serles in $t$ - $t_{0}$, we find that $\bar{v}_{i}(x, t)$ is an infinite functional polynomial series in these quan- 
tities. Here lies the essence of the closure problem. We suspect on intuitional grounds that a simple initial distribution should result in a reasonably simple distribution at later times. Yet we find that the mathematical relation between initial and later distributions is infinitely complicated.

Perhaps the first conclusion suggested by this situation is that our formulation is inappropriate. Perhaps there is a description, other than by moments, in terms of which simple initial distributions would give manifestly simple later distributions. Such a formulation has not been reported, and there is an elementary reason why one should be hard to find. A simple initial distribution, such as the normal one we have adopted, does not mean a simple inftial field $u_{i}\left(x_{m}, t_{0}\right)$ in typical realizations. Even if we make the further requirement that $U_{1 j}\left(x_{m}, t_{0} ; x_{m}^{\prime}, t_{0}\right)$ be a smooth and simple function, a typical realization of $u_{1}\left(x_{m}, t_{0}\right)$ will be a complicated function of $\mathrm{x}$. The solution of the Navier-stokes equation for such realizations is very difficult. And, since the various realizations do not interact, the task is not made easier by considering an ensemble instead of a single system. As long as our formulation of the statistical problem is based ultimately on the Navier-Stokes equation, the quest for a simple, exact statistical solution would seem to depend on finding a simple general solution of the Navier-Stokes equation itself. Let us hope that there is a flaw in this argument?

At this juncture, it is illuminating to compare the turbulence problem with that of the Gibbsian statistical mechanics of Hamiltonian systems [31]. The Gibbs method yields an explicit distribution function for a Hamiltonian system in thermal equilibrium. To obtain this result, 
one need not solve the dynamical equations. We may ask why a similar program cannot be carried out for the turbulence problem.

The answer appears to be two-fold: First, the systems which can be treated successfully by the Gibbs method are spec1al ones. Second, even for these systems, the information obtained in closed form is actually a small part of a complete statistical description; it does not include some types of information which are of interest in turbulence theory. The special property which is central to the Gibbs theory is the existence of Liouvilie's theorem. The latter states that the motion of the "phase cloud" representing an ensemble is incompressible in the phase space of the system; that is to say, it preserves measure. This property depends on the conservative character of the system. In turbulence theory, the phase space is the function space of $\underset{\sim}{\mathrm{V}}$, a correspondence which has been elegantly formulated by Hopf [10]. However, dissipation is important in turbulence, and, as a consequence, measure in the phase space is not even approximately conserved. In the presence of dissipation, the Gibbs method does not lead to closed expressions for distributions, and it remains necessary to solve the equations of motion.

The second part of the answer is that, even for conservative systems in thermal equilibrium, the Gibbs method gives only the simultaneous distribution in closed form. To obtain moments with non-simultaneous time-arguments, one again must return to the equations of motion. If the latter are nonlinear, there arises a closure problem quite analogous to that of turbulence theory. Subordination of the role accorded non-simultaneous averages is not very appropriate in turbulence 
theory because such averages give valuable information about the dynamics of the nonlinear interaction. Moreover, transport phenomena, which always occur when there is dissipation, are most naturally expressed by non-simultaneous averages: Transport implies that something goes from a place at a given time to another place at a later time.

\section{SCXIMMES FOR APPROXIMATE CLOSURE}

The unavailability of a simple method of following exactly the evolution of a simple initial velocity distribution suggests that approximations be sought. This seems particularly appropriate because approximate empirical observations are the real basis for the intultion that simple initial distributions ought to lead to reasonably simple later distributions.

There have been two broad classes of attempts to close off, or circumvent, the infinite set of moment equations by means of approximations. The first consists of essentially phenomenological approaches in which statistical properties, or models of dynamical processes, are postulated on physical grounds. Examples are the universal equilibrtum theory of Kolmogorov [4, 12, 13], Heisenberg's eddy-viscosity theory $[4,9]$, and the maximum-dissipation theory of Malkus [19]. The second class consists of schemes for systematic analytical approximation. Our basic interest in the present paper will be In the mathematical structure of turbulence; consequently, we shall not discuss the first class of approaches. For proper perspective, however, it is highiy important to note that almost all progress to date in the physical understanding of turbulence has come from phenomenological, heuristic theories such as the three cited. It is hardly unfair to say that, so far, the principal achievement of the analytical approaches has been to clarify aspects of their own structures and shortcomings. 
5.1. Isotropic turbulen ve

The andytidul approximations five been arried out principalzy

for the special case ot isotropic or komogeneous turbulence. we shall treat them in this context. but we want flrst to discuss the degree of relevance this artificial problem has to the unlerstanding of actual turbulent flows. The internal aynamical processes which act on the turbulent velocity field $u_{m}(x, t)$ are three: viscous friction, interaction of mean field with turbulent fielz, and selt-intergction of the turbulent fielz. They are representer in $(+.2)$ by the terms inrolving $v,\left[\bar{v}_{m}(x, t) u_{n}(x, t)\right]$, and $\left[u_{m}(x, t) u_{n}(x, t)-U_{m n}\left(x, t ; x_{m}, t\right)\right]$, respectively. only the last of the three processes gives rise to the closure problem, as we have already noted. It is therefore natural to try to understand the problem in the context of isotropic turblilence: There is no mean field, and the selfinteraction of the turbulent field provides the only dymamical process besides the essentially simple one of viscous friction. Moreover, geometrical complications are minimum for this case.

There are two dangers, however, in concentrating on the isotropic case. The first is one of perspective: Even if the closure problem were solved to complete satisfaction, there would still remain, for shear turbulence, the very formidable task of treating the interaction of mean field and turbulent field. The magnitude of this task may be illustrated by the obserration that its most elementary form (valid only for infinitesimal turbulence levels) comprises the laminar stability theory of shear flow [18]. The second danger is that, despite the geometrical simplicity, the problem of finding adequate closure approximations may actually be more difficult for isotropic turbulence than for shear flows. In a shear 
flow, important aspects of the overall dynamics may be dominated by the mean field-turbulent field interaction. If so, cruder approximations to the turbulent field self-interaction may be permissible than in the isotropic problem, where this self-interaction is all-important.

With this preface, let us specialize, in the remainder of this section, to homogeneous, isotropic turbulence, for which $\bar{v}_{i}(x, t)=0$. Then (4.2) reduces to the form

(5.1) $\quad\left(\frac{\partial}{\partial t}-v \nabla^{2}, u_{i}(\underset{\sim}{x}, t)=-\frac{1}{2} P_{i m n}(\nabla)\left[u_{m}(x, t) u_{n}(x, t)\right]\right.$.

Let us assume, as before, that $u_{i}\left(x_{n}, t_{0}\right)$ is multi-variate normal. Let $v_{0}$ be the initial root-mean-square value of any velocity component, and let $\ell_{0}$ be a characteristic correlation length (macro-scale [4]) of the initial covariance $U_{i j}\left(\underset{w}{x}, t_{0} ; x^{\prime}, t_{0}\right)$. In order to exhibit clearly the relative magnitudes of the terms in (5.1), we shall measure lengths and velocities in units $l_{0}$ and $v_{0}$, and times in the unit $l_{0}^{2 / v}$, which is the characteristic time for viscous decay of a velocity structure of scale $\ell_{0}$. Using the new units, which we shall adopt for the remainder of section 5 , we may rewrite (5.1) in the form

(5.2) $\left(\frac{\partial}{\partial t}-\nabla^{2}\right) u_{1}(\underset{m}{x}, t)=-\frac{1}{2} R_{0} P_{i m n}(\nabla)\left[u_{m}(\underset{m}{x}, t) u_{n}(x, t)\right]$,

where

(5.3) $\quad R_{0}=v_{0} l_{0} / v$

is the characteristic Reynolds number for the initial state. 
.2 Expansion in powers of Reynolas number

A formal solution ot'(5.2) may be obtidned iv writine

$(5.4) \quad u_{1}(x, t)=\sum_{n=0}^{\infty} R_{0}^{n} u_{i}(n) \underset{m}{(x, t)}$.

substituting in (5.2), an equating coefficients of like powers of $\mathrm{R}_{0}$ on each side of the equation. In this way we find

(5.5) $\left.\quad u_{i}(0) \underset{\sim}{x}, t\right)=\left[4 \pi\left(t-t_{0}\right)\right]^{-3 / 2} \int \exp \left[-\left|\underset{\sim}{x-x^{\prime}}\right|^{2 / 4}\left(t-t_{0}\right)\right] u_{i}\left(x_{\sim}^{1}, t_{0}\right) d^{3} x^{\prime}$,

and, by iteration, we obtain $u_{i}(n)(\underset{w}{x}, t)$ as a functional power of $u_{1}\left(x_{w}, t_{0}\right)$ of degree $n+1$. The expansion (5.4) then permits us to write any moment of $u_{i}(x, t)$ as an infinite series of linear functionals of the moments $U_{i j}\left(x_{\sim}^{x}, t_{0} ; x^{\prime}, t_{0}\right), U_{i j m}\left(x, t_{0} ; x^{\prime}, t_{0} ; x^{\prime \prime}, t_{0}\right)$, ete. These moments, in turn, all may be expressed in terms of $U_{1, j}\left(\underset{\sim}{x}, t_{0} ; \underset{\sim}{x^{1}}, t_{0}\right)$ according to the assumption that the initial distribution is normal. In this way, we obtain a complete formal solution of the statistical problem. It illustrates our earlier statement that the exact expression of moments at later times in terms of initial moments is one of infinite complexity.

The most straightforward sequence of closure approximations for the present problem consists in truncating the iteration expansions for the moments at successively higher powers of $R_{0}$. The fundumental limitation to this scheme is that we may reasonably expect the approximations to converge rapidly only if $R_{0}<1$, in which case the nonlinear term represents a small perturbation to $(5.2)$ in a typlcal realization. This condition is satisfied during the final period of decay of turbulence produced behind 
a grid. For this case, the zeroth term in the Reynolds number expansion (1.e., pure viscous decay) has been studied by Reissner and by Batchelor and Towsend $[4]$, who obtained interesting results concerning the asymptotic distribution. Several higher terms in the expansion for $U_{i j}$ and $U_{1 j m}$ have been investigated by Deissler $[7,8]$.

For $R_{0} \gg 1$, which is a case of great interest, we cannot expect the iteration expansion to yield adequate approximations. There is an exception, however. For t-to sufficiently small compared to characteristic times of the turbulence, it is reasonable that the expansion should converge well even for $R_{0} \gg 1$. To see this, we note from (5.2) that $\left[\partial^{n} u_{i}(\underset{w}{x}, t) / \partial t^{n}\right]_{t=t_{0}}$ must be a polynomial in $R_{0}$ of degree $n$. Thus, only low powers of $R_{0}$ appear in low orders of a Taylor expansion in $t-t_{0} \cdot A$ similar situation will be examined in section 6.2 .

Although the iteration expansion can be expected to yield useful approximations only for a quite limited range of parameters, it is of great value as an exact formal solution against which to compare approximate solutions obtalned by other means. As we shall point out shortly, several other systematic approximation sequences which have been proposed may be represented formally as summations of contributions selected, according to well-defined rules, from all orders of the iteration expansion.

An interesting question at this point is whether an expansion in powers of $1 / R_{0}$ might be useful for $R_{0} \gg 1$. It seems doubtful that such a procedure would work. The reason is a fundamental asymmetry in the roles of the nonlinear forces and viscous forces. For $R_{0} \ll 1$, the nonlinear forces are a small perturbation. But for $R_{0} \gg 1$, the viscous 
forces are not a small perturbation: The half-lite tor viscous decay of the turbulent kinetic energy is the orjer of the characteristic time $\ell_{0} / \mathrm{r}_{0}$ (original units) for nonlineir effects [4]. This behavior is intimately related to the fact that solutions of the inviscid Navier-stokes equation $\left(R_{0}=\infty\right)$ can develop singular shear-fronts in a finite time, even if the initial conditions are healthy.

5.3 Expansion in cumulants

As we have seen, the iteration expansions for the moments may be derived from a similar expansion for the velocity field in each individual realization. For this reason, one suspects that the 1 teration approximations do not exploit fully the fact that we are interested only in averages. The approximations we shall consider next differ in this respect. They are intrinsically statistical, and they have no meaning as approximations on the individual realizations.

In 1941, Millionstchikov [20] introduced the assumption that fourthorder moments of the distribution of simultaneous velocity amplitudes are related to second-order moments as in a normal distribution. This quasinormality approximation has been applied to isotropic turbulence dynamics by Proudman and Reid [21] and Tatsumi $[27,28]$. A similar approximation for the two-time velocity distribution has been exploited by Chandrasekhar [6] and He1senberg [9]. Hopf [1.] has pointed out that the quasi-nomality approximation is equivalent to retaining only the initial terms in a GramCharlier expansion for the velocity distribution, and he has suggested that a systematic approximation sequence could be based on this expansion. We shall discuss here an approximation sequence based on expansion of the 
velocity moments into cumulants. This is closely related to the GramCharlier expansion.

Let $\mathrm{P}(\mathrm{y})$ be the probability distribution for a single random variable $y$ which has zero mean. Then

(5.6) $\quad \int_{-\infty}^{\infty} P(y) d y=1, \quad\langle y\rangle \equiv \int_{-\infty}^{\infty} y P(y) d y=0$

The cumulants of the distribution may be defined as the coefficients $c_{n}$ In the expansion

$$
\Phi(\alpha)=\exp \left[-\frac{1}{2}\left\langle y^{2}\right\rangle \alpha^{2}+\sum_{n=3}^{\infty} c_{n}(i \alpha)^{n / n j}\right]
$$

where

$$
\text { (5.8) } \Phi(\alpha)=\langle\exp (i \propto y)\rangle=\int_{-\infty}^{\infty} \exp (1 \propto y) p(y) d y
$$

is the characteristic function. Upon evaluating the moments by use of the relation

$$
\text { (5.9) }\left\langle y^{n}\right\rangle=(-i)^{n}\left[d^{n} \Phi(\alpha) / d \alpha^{n}\right]_{\alpha=0} \text {, }
$$

we find

$$
\begin{aligned}
& \left\langle y^{3}\right\rangle=c_{3}, \\
& \left\langle y^{4}\right\rangle=3\left\langle y^{2}\right\rangle^{2}+c_{4}, \\
& \left\langle y^{5}\right\rangle=10\left\langle y^{2}\right\rangle c_{3}+c_{5}, \\
& \left\langle y^{6}\right\rangle=15\left\langle y^{2}\right\rangle^{3}+10 c_{3}^{2}+15\left\langle y^{2}\right\rangle c_{4}+c_{6},
\end{aligned}
$$


The significance of the cumulants is that they measure the degree and comDlexity of the deviation of $\mathrm{P}(y)$, and its moments, frum a normal distribution.

If an expansion analogous to $(5.7)$ is made for the characteristic functional [10.11] of the one-time velocity distribution, a sequence of abnroximations to the various moments can be formed by tmancating the expansion so as to admit successively higher cumulants. In the first nontrivial approximation, we admit third-order cumulants only. Then [in correspondence to the second of relations $(5 \cdot 10)$. with $c_{4}=0$ ] we find

$$
\begin{aligned}
& U_{1 j m n}\left(\underset{\sim}{x}, t ; x^{\prime}, t ; x_{\sim}^{\prime \prime}, t ; x^{\prime \prime}, t\right)=U_{i j}\left(\underset{\sim}{x}, t ; x^{\prime}, t\right) U_{m n}\left(x_{m}^{\prime \prime}, t ; x^{\prime \prime \prime}, t\right) \\
& +U_{1 m}\left(\underset{\sim}{x}, t ; x_{n}^{\prime \prime}, t\right) U_{j n}\left(\underset{\sim}{x^{\prime}}, t ; \underset{\sim}{x^{\prime \prime \prime}}, t\right)+U_{i n}\left(\underset{\sim}{x}, t ; \underset{\sim}{x^{\prime \prime \prime}}, t\right) U_{j m} \underset{m}{\left(x^{\prime}, t ; x^{\prime \prime}, t\right)},
\end{aligned}
$$

which is the quasi-normality approximation employed by Proudman and Reid [21] and Tatsumi [27,28]. More generally, by cutting off the expansion after the cumulant of nth order, we obtain a closed expression for moments of order $n+l$ in terms of moments of lower orders. We shall call the sequence of approximations so obtained the cumulant-discard approximations.

Equation (5.11) may be used to close the moment equations as follows. From (5.3) we form equations for the total time-derivatives of $\mathrm{U}_{i j}\left(\underset{\sim}{\mathrm{x}}, \mathrm{t} ; \mathrm{x}_{\sim}^{\prime}, t\right)$ and $\mathrm{U}_{i j \mathrm{k}}\left(\underset{\sim}{\mathrm{x}}, \mathrm{t}: \underset{\sim}{\mathrm{x}^{\prime}}, t ; \mathrm{x}_{\sim}^{\prime \prime}, \mathrm{t}\right)$. We then eliminate the fourth-order moments from these equations by (5.11), thereby obtaining a closed set of simultaneous equations for $U_{i j}\left(\underset{\sim}{x}, t ; x_{\sim}^{\prime}, t\right)$ and $U_{i j k}\left(\underset{\sim}{x}, t ; \underset{\sim}{x^{\prime}}, t: \underset{\sim}{x^{\prime \prime}}, t\right)$. The Third-order moments can be eliminated from this set by integration so that, finally, we end up with an integro-differential equation for $U_{i j}\left(\underset{\sim}{x}, t ; x_{m}^{\prime}, t\right)$ alone. 
At the next level, we eliminate fifth-order moments by means of the second cumulant-discard approximation [corresponding to the third of relations (5.10), with $\left.c_{5}=0\right]$. We thereby obtain simultaneous equations for second-, third-, and fourth-order moments. Then we eliminate the fourthorder moments by integration and finally obtain closed, simultaneous equations for $U_{i j}\left(\underset{\sim}{x}, t ; x_{\sim}^{\prime}, t\right)$ and $U_{i j k}\left(\underset{\sim}{x}, t ; \underset{\sim}{x^{\prime}}, t ; \underset{\sim}{\prime \prime}, t\right)$. As we go up in this sequence of approximations, the complexity of the final set of equations which must be solved increases very rapidly. The isotropy condition results in substantial simplifications, however.

The cumulant-discard approximations bear a definite relation to the iteration expansion. If we solve the final equations for the nth cumulantdiscard approximation by expansion in powers of $R_{0}$, we find that the expansion for $U_{i j}\left(\underset{\sim}{x}, t ; x^{\prime}, t\right)$ contains all (even) powers of $R_{0}$. It agrees precisely with the exact iteration expansion to order $\mathrm{R}_{0}^{2 \mathrm{n}}$. This is a consequence of the fact that the exact iteration expansion of the cumulant of order $n+2$ starts with $R_{0}^{n}$. Thus, the neglect of this cumulant can affect only terms above a certain order in the expression for $U_{i j}$. For powers of $R_{0}$ greater than $2 n$, the cumulant-discard result for $U_{i j}$ is no longer exact, but the coefficient-function of each power of $\mathrm{R}_{0}$ bears a definite relation to that in the exact expansion. The coefficient in the exact expansion consists of a sum of individual terms, and that in the cumulant-discard result consists of only some of these terms, selected according to well-defined and simple rules. We shall discuss this relation in more detail, for a simpler problem, in section 6.5 .

Since the nth cumulant-discard approximation to $U_{i j}$ agrees with the exact iteration expansion to order $R_{0}^{2 n}$, 
it is clear that for $R_{c} \cdots 1$, or for t-t, sufficiently small, this epproximation will be indistineuistable from the nth iteration approxintion. of more interest is the behavior ot the cumulant-aiscarapproxirations for $\mathrm{R}_{0} \gg 1$. The fact that they contain all powers of $\mathrm{R}_{\mathrm{g}}$ offers the hope that they may converge rapialt when an expansion in $R$, does not. It is not clear, at the present time, whether this is the case or not. When $R_{0}$ is large, it seems unlikely that all the cumulants can be small, but this does not exclude the possibility that their magnitude. or aynamical importance, may decrease with increasing order. If this does happen, with sufficient rapidity, it is reasonable to expect good convergence. The existing experimental evidence on the magnitude of cumulants [4] is difficult to interpret. This is partly because of low accuracy. but the principal reason is that the appropriate quantitative theoretical criteria for "smallness" have not been worked out. Apart from the question of the quantitative accuracy of the cumulantdiscard approximations. there is a danger that they may lead to unphysical consequences of a disconcerting kind at high $\mathrm{R}_{\mathrm{O}}$. This arises because the distribution functionals obtained by truncation of the expansion in cumulants are, in general, not positive-definite in the function-space of $\mathbf{m}_{m}(\underset{\sim}{x}, t)$ : that is to say, the cumulant-discard approximations can lead to negative probabilities. This seems actually to occur in the first approximation. Proudman and Reid [2l] have been able to integrate exactly the equation for the skewness factor $S=\left\langle u_{1}^{3}\right\rangle /\left\langle u_{1}^{2}\right\rangle^{3 / 2}$ in this approximation. Their result shows that $S$ takes values which, according to inequalities derived by Betchov [5], are incompatible with a positive-definite distribution having zero fourth-order cumulants. 
The generation of negative probabilities raises the possibility that the cunulant-discara approximations may lead to non-positive-definite wavenumber spectra $E(k)$ for the kinetic energy. In inhomogeneous turbulence, they may lead to negative kinetic energy density $\left\langle\frac{1}{2}[u(x, t)]^{2}\right\rangle$ in some regions of space. The cumulant-discard approach so far has been explicitly worked out only for the first approximation and only for isotropic turbulence. The equations in this case have been integrated to give the evolution of $E(k)$ only for very short times or small $R_{0}$, where the validity of the approximations is not in doubt. Thus, the questions we have raised are unanswered at present. However, as we shall show in section 6.3, the cumulant-discard approximations for the one-time distribution do give unphysical behavior when they are applied to the convection of a passive scalar field $\psi(x, t)$ by a random velocity field. They lead there to a situation in which $\left\langle[u(x, t)]^{2}\right\rangle$, which is analogous to mean kinetic energy density. goes negative in places and $\int\left|\left\langle[\psi(\underset{\sim}{x}, t)]^{2}\right\rangle\right| a^{3} x$, which should be conserved, increases without limit. The difficulties with negative probabilities become demonstrably severe in turbulence dynamics proper, if the cumulant-discard approach is extended to the many-time distribution so as to give non-simultaneous as well as simultaneous averages [14]. The existence of all these troubles is discouraging because the cumulant-discard approach seems to be a very natural and logical one. It has, moreover, a record of successes in other physical problems.

\subsection{Approximation by stochastic models}

An alternative to the iteration and cumulant-discard approaches has recently been proposed by the author [16]. The basic idea is to replace the 
true dyramical problem : a molel in which fictitius couplings ane tntrcduced among an infinite oollection cf flow systems. The true problem then is recovered as the limit in which all the couplints vanish. The prasings of the couplings change at random as we go from one individual system ir the collection to another. Thus. tre model involves a new stochastic element which is absent in the true problem.

The aditional randomess plays a paradoxical role. It makes the detailed dynamies of the collection of coupled systems infinitely more complicated. But it vastly simplifies the moment equations obtained by averaging over an ensemble of realizations of the collection. This is because cancellation effects. arising from the random phasings of the couplings, eliminate many of the complexities which the nonlinearity of the dynamical processes would otherwise produce. As a result, the model problem leads, without any approximation. to closed equations for moments of low order. in contrast to the infinite set of coupled equations which characterize the true problem. We noted in section 4 that the consideration of an ensemble could not, in itself, be expected to alleviate the fundamental difficulty of solving the Navier-Stokes equation in the realizations which make up the ensemble. In the model method, we circumvent this limitation by making many systems interact with each other in a stochastic fashion.

Successive approximations in the stochastic model scheme must be sought by constructing a sequence of models in which the random dynamical couplings are systematically reduced. This must be done in such a fashion that exact closed equations (which will be of increasing complexity) result at each stage. So far, only one model has been explicitly constructed. A 
putative second model has been investigated, but its self-consistency has not been proved. At present, we cannot be sure that there actually exists an infinite sequence of models which converge to the true problem. The stochastic model scheme has the advantage that negative crobability troubles, such as negative spectra or energy-densities, cannot arise. This is because the final equations are the exact description of the dynamical model and do not involve any approximation to its probability distribution. A substantial price is paid for this advantage, however. The first approximation in the cumulant-discard sequence for isotropic turbu-

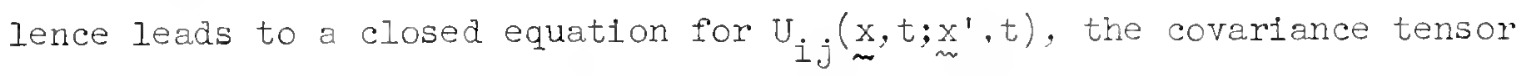
for equal times. In the first stochastic model also, closed equations are obtained for the coveriance tensor. However, it is necessary to determine the full non-simultaneous tensor $U_{i j}\left(x, t ; \underset{\sim}{\prime}, t^{\prime}\right)$. The simultaneous-argument problem cannot be separated off. At the same time it is necessary to solve equations which determine the average response of the system to infinitesimal disturbances. This adaitional information is actually of considerable interest (cf. Sections 2 and 4), but the equations necessarily are more complex.

If the result for $\mathrm{U}_{i j}\left(\underset{\sim}{\mathrm{x}}, \mathrm{t} ; \mathrm{x}_{\sim}^{\mathrm{x}}, t\right)$ in the first stochastic model is expanded in powers of $R_{0}$, a definite relation is found to the exact iteration expansion for $U_{i j}\left(\underset{\sim}{x}, t ; \underset{\sim}{x^{1}}, t\right)$. In common with the first cumulant-discard approximation, the model result contains all (even) powers of $R_{0}$ and is identical with the iteration expansion to order $\mathrm{R}_{0}^{2}$. The coefficients of the higher powers of $R_{0}$ represent a selection of terms from the corresponding orders of the iteration expansion, but a more comprehensive one than that Included in the first cumulant-discard approximation. The selection rules are given in [16]. 
When applied to isotroplc turbulence, the furst stochustic model may be given an altemative interpretation which does not involve the coupling̈ of a collection of flow systems. It may be regarded as an approximation on the dynamical interaction of the spat1al Fourier amplitudes of the velocity field in a single (infinite) system [15]. The approximation consists of retaining only that contribution, to the phase correlation of each triad of Fourier amplitudes, which is induced by the direct dynamical Interaction of the three amplitudes.

The model gives a description of isotropic turbulence dynamics which appears to be qualitatively acceptable [15]. It prealcts separate energycontaining, inertial, and dissipation ranges at high Reynolds numbers. In adation, 1t yields plausible statistical stability properties. However, the asymptotic spectmu law for the inertial range disagrees slightly with that of the Kolmogorov theory, and it is probably inaccurate to that extent. It is not yet known how the inertial range structure is altered in the tentative second model.

\section{AN APPIICATION OF THE CLOSURE APPROXIMATIONS}

\subsection{Convection by a random velocity field}

We shall now illustrate some properties of the several closure schemes by applying them to a problem for which they all yield explicit results that can be compared with the exact solution. For this purpose, we consider the convection of marked fluid particles by a prescribed random velocity field. Since the initial analytical treatment of this problem by Taylor [29]. It has received extensive study. The formulation we shall employ here follows that of Batchelor $[1-3]$. 
Consider an ensemble of incompressible flows in which the velocity field $\underset{\sim}{u(x)}$ has an isotropic, multivariate normal distribution, and is independent of time in each realization. The distribution then is wholly characterized by the covariance

$$
\text { (6.1) } \quad U_{i j}\left(\underset{\sim}{x-x_{m}^{\prime}}\right)=\left\langle u_{1}(\underbrace{(x)}_{n} u_{j}(\underbrace{\prime}_{\sim})\rangle\right. \text {. }
$$

A physical embodiment of a typical reallzation could be a vessel of water agitated at low Reynolds number by a very large number of small, steady stirring devices, located and orlented at random throughout the fluid. (The stirring devices might, for example, be ciliated micro-organisms.) The concentration of marked particles $\psi(\underset{\sim}{x}, t)$ obeys the equation $(6.2)$

$$
\left(\frac{\partial}{\partial t}-k \nabla^{2}\right)_{\psi}=-u_{i} \frac{\partial \psi}{\partial x_{i}}
$$

where $k$ is the molecular diffusivity. We shall treat the simplest statistical problem associated with this equation: Given the initial mean concentration $\langle\psi(\underset{w}{x}, 0)\rangle$, with $\psi(\underset{\sim}{x}, 0)$ statistically independent of $\underset{\sim}{u}$, find the lat,er mean concentration $\langle\psi(x, t)\rangle$. Since (6.2) is linear, this problem reduces tc that of finding the average Green's function, defined by

$$
\text { (6.3) } G\left(\underset{m}{x-x_{m}^{1}}, t\right)=\langle\psi(\underset{w}{x}, t)\rangle, \quad \psi(\underset{m}{x}, 0)=\delta^{3}\left(\underset{\sim}{x-x_{m}}\right) \text {, }
$$

where $\psi(\underset{w}{x}, t)$ obeys $(6.2)$ and $\delta^{3}(\underbrace{x}_{w}-x_{\sim}^{\prime})$ is the three-dimensional Dirac function. $G\left(\underset{w}{x-x^{2}}, t-t^{\prime}\right) d^{3} x$ is the probability that a marked particle introduced at $\left(\underline{\sim}^{\prime}, t^{\prime}\right)$ is found in the volume $d^{3} x$ at $(\underset{w}{x}, t)$. 
If we multiply (6.2) by various powers of $\psi$ and average, wes find a closure problem quite stmilar to that of turbulence dynamics. This occurs because (6.2) is nonlinear in stochastic quantities, although it is linear in the dynamic variable. We shall apply to the determination of $G(x, t)$ all three of the closure schemes discussed in section 5 . First, we wish to establish some features of the exact solution by appealing to simple analytical and physical arguments.

As in Section 5 , let $\ell_{0}$ and $v_{0}$ be the correlation length and rootmean-square velocity component associated with $\underset{n}{\mathrm{u}}(\underset{\sim}{\mathrm{x}})$. The parameter which plays the role of $R_{0}$ is

$$
B_{0}=\ell_{0} v_{0} / k \cdot \quad 3
$$

We shall confine our attention to the case $B_{0} \gg 1$. (We shall not adopt special time, length, and velocity scales here as we did in Section 5)

Two asymptotic ranges may be distinguished in which $G(x, t)$ has a simple form. They are $t \ll \ell_{0} / v_{0}$ and $t \gg \ell_{0} / v_{0}$. In the first range, the distance from the space origin travelled by almost all particles is $\ll \ell_{0}$. Thus, they have very nearly their initial velocity $\underset{\sim}{u}(0)$. The distribution is then the resultant of two independent Gaussian processes: molecular diffusion and a Gaussianly distributed displacement tư( $(0)$. It follows that

$$
\text { (6.5) } \begin{aligned}
G(\underset{\sim}{x}, t)=\left[4 \pi\left(k t+\frac{1}{2} v_{0}^{2} t^{2}\right)\right]^{-3 / 2} & \exp \left[-|\underset{\sim}{\mid x}|^{2} /\left(4 k t+2 v_{0}^{2} t^{2}\right)\right] \\
& \left(t<\ell_{0} / v_{0}\right),
\end{aligned}
$$

3 If $\psi(x, t)$ represented temperature fluctuation instead of marked particle concentration, and $k$ were kinematic heat conductivity, then $\mathrm{B}_{\mathrm{O}}$ would be called a Peclét number. 
and that

(6.6) $\left.\left\langle x_{1}{ }^{2}\right\rangle=\int x_{1}{ }^{2}{ }_{G} \underset{\sim}{x}, t\right) d^{3} x=2 k t+v_{0}{ }^{2} t^{2} \quad\left(t<<\ell_{0} / v_{0}\right)$,

where $x_{1}$ is any component of displacement. We see from (6.5) and (6.6) that molecular afffusion is negligible for most times if $\mathrm{B}_{0} \gg 1$, but that it dominates for very short times $t \ll k / v_{0}^{2}=B_{0}^{-1} l_{0} / v_{0}$.

For $t \gg \ell_{0} / v_{0}$, we may anticipate that the convection acts as a random walk process with a step length approximately $\ell_{0}$ and an effective velocity approximately $v_{0} \cdot{ }^{4}$ Hence, we shall assume that $G(\underset{m}{x}, t)$ has a Gausian form in this range also:

(6.7) $\quad G(x, t)=\left(4 \pi a v_{0} \ell_{0} t\right)^{-3 / 2} \exp \left[-\left|x_{m}^{x}\right|^{2} / 4 \mathrm{av}_{0} l_{0} t\right], \quad\left(t \gg l_{0} / v_{0}\right)$,

(6.8) $\left\langle x_{1}^{2}\right\rangle=2 \mathrm{av}_{0}{ }^{\ell}{ }_{0}$, $\left(t \gg \ell_{0} / v_{0}\right)$

Here a is a number fixed by the shape of $U_{i j}\left(\underset{\sim}{\left(x-x^{\prime}\right.}\right)$, and we neglect molecular diffusion completely.

On the basis of (6.5)-(6.8) we shall distinguish three asymptotic time ranges

$$
\begin{array}{ll}
\text { Very short times: } & t \ll \mathrm{B}_{0}^{-I_{\ell} / v_{0}} \\
\text { Short times: } & \mathrm{B}_{0}{ }^{-I_{\ell} / v_{0}}<t<\ell_{0} / \mathrm{v}_{0} \\
\text { Long times: } & t \gg \ell_{0} / v_{0}
\end{array}
$$

The three ranges will be distinct if $B_{O}$ is large enough.

4 For a static velocity field, the asymptotic correspondence of random convection to a random walk does not appear to hold in two-dimensional flows. 
6.2 Iteration expansior

Equation (6.2) may be solved fornally by iteration, in analogy to the procedure discussel in section .2 . If the result 1 s averaged over the normal alstribution of $\underset{\sim}{u}(\underset{\sim}{x})$, we find

$(6.9)$

$$
\begin{aligned}
G(\underset{\sim}{x}, t) & =G_{0}(x, t)+\int_{0}^{t} d s \int_{0}^{s} d s^{\prime} \int a^{3} y \int d^{3} y^{\prime} G_{0}(x-y, t-s) \\
& \times U_{i j}\left(y_{\sim}-y_{\sim}^{\prime}\right) \frac{\partial G_{0}\left(y_{\sim}^{y-y^{\prime}}, s^{\prime}-s^{\prime}\right)}{\partial y_{i}} \frac{\partial G_{0}\left(y_{\sim}^{\prime}, s^{\prime}\right)}{\partial y_{j}^{\prime}}+\ldots,
\end{aligned}
$$

where

(6.10) $\quad G_{0}(x, t)=(4 \pi k t)^{-3 / 2} \exp \left(-|x|^{2} / 4 k t\right)$

is the solution of the homogeneous equation with $\underset{\sim}{u}(\underset{w}{x})=0$. Equation (6.9) also may be written as the differential equation

$$
\begin{aligned}
& \left(\frac{\partial}{\partial t}-k \nabla^{2}\right) G(x, t)=\int_{0}^{t} d s \int d^{3} y U_{i j} \underset{\sim}{(x-y)} \\
& \times \frac{\partial G_{0}(x-y, t-s)}{\partial x_{1}} \frac{\partial G_{0}(y, s)}{\partial y_{j}}+\ldots, \quad G(\underset{\sim}{x}, 0)=\delta^{3}(x) .
\end{aligned}
$$

The higher terms, which are not shown explicitly in (6.9) and (6.11), Involve derivatives of $U_{1 j}$ as well as $U_{1 j}$ itself. If we were to adopt approprlate characterlstic untts, as was done in Section 5, we would see that (6.9) is an expansion in powers of $B_{0}^{2}$ (odd powers of $B_{0}$ do not appear). 
For $t \ll \ell_{0}^{2} / k=B_{0} \ell / v_{0}$, which can include all three of our asymptotic time ranges $1 f B_{0}$ is large, $G_{0}(x, t)$ is negligible unless $|x|<\ell_{0}$. Then we may take $U_{1 j}\left(y_{m}-y_{m}^{\prime}\right) \approx U_{1 j}(0)=\delta_{i j} v_{0}^{2}$ in $(6.9)$, retain only the explicitly shown terms, and thereby obtaln

$$
G(\underset{\sim}{x}, t)=G_{0}(x, t)+\frac{1}{2} v_{0}^{2} t^{2} \nabla^{2} G_{0}(x, t)
$$$$
\left(\mathrm{t} \ll \mathrm{B}_{0}^{\ell} \mathrm{O}_{\mathrm{O}} / \mathrm{v}_{\mathrm{O}}\right)
$$

$$
\left\langle x_{1}^{2}\right\rangle=2 k t+v_{0}^{2} t^{2}
$$

$$
\left(t \ll B_{O} \ell_{0} / v_{0}\right)
$$

as the first iteration approximation. 5 For $t \ll \mathrm{B}_{0}^{-1} \ell_{0} / \mathrm{v}_{0}$, the second terms on the right sides of (6.12) and (6.13) are small corrections, and the iteration approximation is a good one. For longer times, (6.12) provides a very poor approximation to (6.5) and (6.7). It indicates that $G(x, t)$ is negligible for $|x| \gg(k t)^{1 / 2}$, whereas, according to (6.5) or (6.7), it should be spread over the much greater range $|x| \sim v_{0} t$ or $|x| \sim\left(\operatorname{av}_{0} \ell_{0} t\right)^{1 / 2}$. Moreover, $G(\underset{\sim}{x}, t)$ is strongly negative in a region $|x| \lesssim(k t)^{1 / 2}$ surrounding the origin, in contradiction of the physical requirement that the probability of finding a particle be non-negative. It is of interest, however, that (6.13) agrees with the exact solution throughout the short time range $t<\ell_{0} / v_{0} \cdot 6$

We wish now to examine the convergence properties of the full iteration expansion, for $\mathrm{B}_{0} \gg 1$. The expansion can be generated very simply in the short time range if we rewrite (6.5) in operational form and then

5 To obtain (6.12) we perform partial integrations and note that $G_{0}$ is Its own convolution in space-time.

6 The fact that $\left\langle x_{1}{ }^{2}\right\rangle$ grows properly $\left(t<\ell_{0} / v_{0}\right)$, although $G(x, t)$ remains concentrated near the origin, is related to the indefiniteness of $G(x, t)$. Although the total probability is conserved, the total probability in the region where $G(x, t)$ is positive grows with $t$. 
expand in powers of $\mathrm{v}_{0}$. We have

$(6.14) \quad G(x, t)=\exp \left(\frac{1}{2} v_{0}^{2} t^{2} \nabla^{2}\right) G_{0}(x, t)$

$$
\begin{aligned}
= & {\left[1+\sum_{n=1}^{\infty}\left(\frac{1}{2} v_{0}^{2} t^{2} \nabla^{2}\right)^{n} / n !\right] G_{0}(x, t) } \\
& \left(t<l_{0} / v_{0}\right) .
\end{aligned}
$$

It can be seen from (6.14) that successive terms in the expansion display more and more complicated oscillations in slgn. For $B_{0} \gg 1$, the successive terms grow in magnitude, except for very short times. Thus, the iteration approximations to $G(x, t)$ do not form a usefully convergent sequence. We should note, however, that $\left\langle x_{1}{ }^{2 m}\right\rangle$ is given correctly for $m \leq N$, in the short time range, if $(6.14)$ is truncated after the term $\mathrm{n}=\mathrm{N}$.

The actual radius of convergence of (6.14) is easy to determine. If we examine the right side of (6.5) as a function in the complex $v_{0}$ plane, wee that $1 t$ is analytic at the origin but has essential singularittes at $v_{0}= \pm i(2 k / t)^{1 / 2}$. Hence, the domain of convergence of $(6.14)$ as a power series in $\mathrm{v}_{\mathrm{O}}$ is given by

$$
t<2 k / v_{0}^{2}=2 B_{0}^{-1} l_{0} / v_{0}
$$

In the case $B_{0} \ll 1$, which we have not considered, this result suggests that the domain of convergence extends into the long time range $t \gg l_{0} / v_{0}$. However, (6.5) is not valid there. Actually the 1 teration expansion for $G(x, t)$ appears to converge for all $t$, if $B_{0} \ll 1$. 


\subsection{Cumulant-discard approximations}

In analogy to Section 5.3, we may construct a sequence of approximations to $G(\underset{\sim}{x}, t)$ by truncating a cumulant expansion of the joint distribution of $u(\underset{m}{x})$ and $\psi(\underset{\sim}{x}, t)$. This has been carried out for a more general case by Roberts [24]. The sequence of closures relevant to the present problem is

$$
\left\langle u_{i}\left(\underset{\sim}{x^{\prime}}\right) u_{j}\left(x_{m}^{\prime \prime}\right) \psi(\underset{\sim}{x}, t)\right\rangle=U_{i j}\left(\underset{\sim}{x^{\prime}}-x_{m}^{\prime \prime}\right)\langle\psi(\underset{\sim}{x}, t)\rangle,
$$

$(6.15)$

$$
\begin{aligned}
& \left\langle u_{i}\left(x_{\sim}^{\prime}\right) u_{j}\left(x_{\sim}^{\prime \prime}\right) u_{m}\left(\underset{\sim}{x^{\prime \prime \prime}}\right) \psi(\underset{\sim}{x}, t)\right\rangle=U_{i j} \underset{\sim}{\left(x^{\prime}-x_{m}^{\prime \prime}\right)}\left\langle u_{m}\left(x_{\sim}^{\prime \prime \prime}\right) \psi(\underset{\sim}{x}, t)\right\rangle \\
& \left.+U_{i m}\left(x_{m}^{\prime}-x^{\prime \prime \prime}\right)\left\langle u_{j}\left(x_{\sim}^{\prime \prime}\right) \psi(\underset{\sim}{x}, t)\right\rangle+U_{j m} \underset{\sim}{\left(x^{\prime \prime}-x_{\sim}^{\prime \prime \prime}\right.}\right)\left\langle u_{i}\left(x_{\sim}^{\prime}\right) \psi(\underset{\sim}{x}, t)\right\rangle,
\end{aligned}
$$

By using the first of these equations to close off the pair of moment equations

$$
\left\langle\frac{\partial}{\partial t}-k \nabla^{2}\right)\langle\psi(\underset{\sim}{x}, t)\rangle=-\left\langle u_{i} \underset{\sim}{x} \frac{\partial \psi(\underset{\sim}{x}, t)}{\partial x_{i}}\right\rangle,
$$

$$
\left(\frac{\partial}{\partial t}-k \nabla_{x}^{2}\right)\left\langle u_{i}\left(x_{\sim}^{\prime}, \underset{\sim}{(x, t)}\right\rangle=-\left\langle u_{i} \underset{\sim}{\left(x^{\prime}\right)} u_{j} \underset{\sim}{(x)} \frac{\partial \psi(x, t)}{\partial x_{j}}\right\rangle,\right.
$$

we obtain, after manipulation, the first cumulant-discard approximation

$$
\begin{aligned}
& \text { (6.18) }\left(\frac{\partial}{\partial t}-k \nabla^{2}\right) G(x, t)=\int_{0}^{t} d s \int a^{3} x^{\prime} U_{1 j}\left(\underset{\sim}{x-x_{\sim}^{\prime}}\right) \\
& x \frac{\partial G_{0}\left({\underset{w}{w}}_{w}, t-s\right)}{\partial x_{1}} \frac{\partial G\left(x_{w}^{x^{\prime}}, s\right)}{\partial x_{j}^{\prime}}, \quad G(\underset{w}{x}, 0)=\delta^{3}(\underset{\sim}{x}) .
\end{aligned}
$$


It w1I be noted that (6.18) bears a resemblance to the first 1terat1on approximation, as given by the explicitly shown terms in (6.11). Equation (6.18), however, is an homogeneous, linear, integro-differential equation instead of an inhomogeneous, linear, differential equation.

For $t \ll B_{0} l / v_{0},(6.18)$ may be reduced to the form

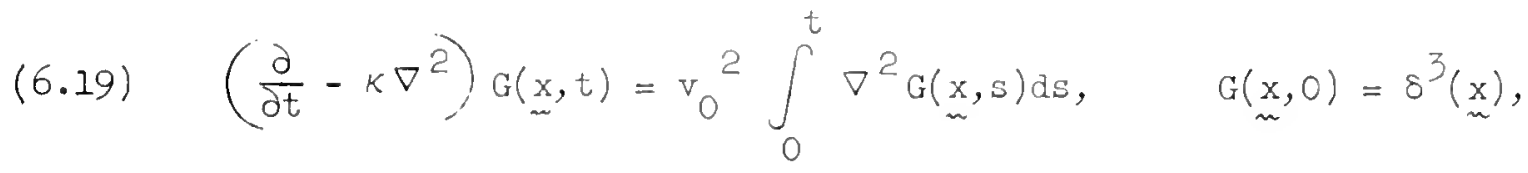
of which the solution is

$$
\begin{aligned}
& G(\underset{\sim}{x}, t)=\exp \left(k \nabla^{2} t\right)\left[-\frac{I}{4 \pi|\underset{\sim}{\mid x}|} \frac{\partial}{\partial|\underset{\sim}{\mid x}|} \varepsilon\left(|\underset{\sim}{\mid x}|-v_{0} t\right)\right] \\
& =-(4 \pi k t)^{-3 / 2} \frac{k t}{\mid \underset{\sim}{|x|} \frac{\partial}{\partial|x|}}\left\{\exp \left[-\frac{\left(||_{\sim}^{x} \mid-v_{0} t\right)^{2}}{4 k t}\right]+\exp \left[-\frac{\left(|x|_{\sim}+v_{0} t\right)^{2}}{4 k t}\right]\right\} .
\end{aligned}
$$

For $t \ll B_{O}^{-1} l_{0} / v_{0}$, the range where the f'irst iteration approximation (6.12) Is val1d, 1t may be verifled that (6.20) reduces to (6.12), as would be anticipated by arguments simflar to those in section 5.2. For longer times, where the first iteration approximation is poor, (6.20) also is a poor approximation to $(6.5)$ and $(6.7)$. For such times, (6.20) gives to $G(\underset{w}{x}, t)$ the form of an outgoing spherical wave-front, consisting of a narrow positive crest $\left[\right.$ width $\left.\sim(k t)^{I / 2}\right]$ followed by a similar negative crest, which moves outward at a constant velocity $v_{0}$. Thus it gives negative probabilities, as did the iteration approximation. The behavior of the present approximation is somewhat better, however, in that $\mathrm{G}(\mathrm{x}, \mathrm{t})$ at least 
does not remain concentrated about the orlgin. Equation (6.20) gives for $\left\langle x_{1}^{2}\right\rangle$ the result (6.13) obtained in the first 1teration approximation. As we have noted, this expression 1 s correct for $t \ll \ell_{0} / v_{0}$ but not for $t \gg \ell_{0} / v_{0}$.

The next few approximations in the cumulant-discard sequence are not difficult to work out in the short time range $t<<\ell_{0} / v_{0}$. We shall give the final results for the second and third approximations. Using the second of equations (6.15) to close off the flrst three equations in the moment sequence which commences with (6.16) and (6.17), one finds

(6.21) $G(\underset{m}{x}, t)=\exp \left(k \nabla^{2} t\right)\left[\frac{I}{6 \pi|\underset{n}{\mid x}|^{2}} \delta\left(\left|x_{n}^{x}\right|\right)-\frac{1}{12 \pi|\underset{m}{\mid}|} \frac{\partial \delta\left(|\underset{n}{x}|-\sqrt{3} v_{0} t\right)}{\partial|\underset{\sim}{x}|}\right]$. The third cumulant-discard approximation is

$$
\text { (6.22) } \begin{aligned}
G(\underset{\sim}{x}, t)=-\frac{I}{24 \pi} \exp \left(\kappa \nabla^{2} t\right) & \left\{(3+\sqrt{6})|\underset{\sim}{\mid x}|^{-1} \frac{\partial \delta\left[|x|-(3-\sqrt{6})^{1 / 2} v_{0} t\right]}{\partial|\underset{\sim}{x}|}\right. \\
& \left.+(3-\sqrt{6})|\underset{\sim}{x}|^{-1} \frac{\partial \delta\left[|\underset{\sim}{x}|-(3+\sqrt{6})^{1 / 2} v_{0} t\right]}{\partial|x|}\right\} .
\end{aligned}
$$

It is apparent that the unphysical characteristics of wave-like propagation and negative probabilities persist in these higher approximations. However, there is a kind of improvement which also characterized the higher iteration approximations: The quantity $\left\langle x_{1}{ }^{2 m}\right\rangle$ is given correctly for $\mathrm{m} \leqq N$ in the $N$ th cumulant-discard approximation, provided $t \ll \ell_{0} / v_{0}$. For $t \gg \ell_{0} / v_{0}$, the behavior of the second and third cumulant-alscard approximations is complicated, but, like the first one, they do not appear to go 
over into the random walk behavior described by (6.7) and (6.8). Instead, they appear to yleld $\left\langle x_{1}^{2}\right\rangle \propto t^{2}$ in this time ranee.

The results we have cited seem clearly to indicate that ne1ther the magnitude nor the importance of the cumulants of the joint distribution of $\underset{\sim}{u}$ and $\psi$ decreases with increasing order when $\mathrm{B}_{0} \gg 1$. Apart from the question of the quantitative accuracy with which the cumulant-discard approximations represent $G(\underset{\sim}{x}, t)$, it is disquieting that they lead to negative probabllities for finding a particle.

Similar unphysical results occur, and are more disconcerting, if we use the cumulant-discard approximations to trace the evolution of the manifestly positive-definite quantity $\left\langle[\psi(x, t)]^{2}\right\rangle$. We shall outline this analysis for the case of pure convection $(k=0)$. In this case, $\int[\psi(\underset{\sim}{x}, t)]^{2} a^{3} x$, which plays a role analogous to total kinetic energy in turbulence dynamics, is conserved.

Let us take

$$
\psi(\underset{w}{x}, t)=\bar{\psi}+\psi^{\prime}(\underset{w}{x}, t),
$$

where $\bar{\psi}$ is a constant background concentration and $\psi^{\prime}(\underset{\sim}{x}, t)$ is a fluctuation which satisfies $\left\langle\psi^{\prime}(x, t)\right\rangle=0$ [a condition preserved by $\left.(6.2)\right]$. We wish to trace the evolution of

$$
\left.\Psi(\underset{m}{x}, t)=\left\langle\left[\Psi^{\prime} \underset{\sim}{x}, t\right)\right]^{2}\right\rangle
$$

under the condition that $\psi^{\prime}(\underset{m}{x}, 0)$ is statistically independent of $u$. From (6.2), with $k=0$, we have

$$
\frac{\partial}{\partial t}\left[\psi^{\prime}(\underset{m}{x}, t)\right]^{2}=-u_{i} \underset{\sim}{(x)} \frac{\partial}{\partial x_{i}}\left[\psi^{\prime}(\underset{\sim}{x}, t)\right]^{2},
$$


a linear equation identical with that obeyed by $\underset{\sim}{(x, t)}$ itself. The sequence of cumulant-discard closures appropriate to the present problems are identical with $(6.15)$ if we replace $\psi(\underset{m}{x}, t)$ there by $\left[\psi^{\prime}(x, t)\right]^{2}$. Thus the first closure is 7

(6.26) $\left\langle u_{i}\left(x_{m}^{\prime}\right) u_{j}\left(\underset{m}{x^{\prime \prime}}\right)\left[\psi^{\prime}(\underset{m}{x}, t)\right]^{2}\right\rangle=U_{1 j} \underset{x}{\left(x^{\prime}-x_{m}^{\prime \prime}\right) \Psi(x, t)}$.

It follows immediately from these simflarities that the cumulant-discard approximations to $\Psi(\underset{\sim}{x}, t)$ and $G(\underset{\sim}{x}, t)$ are related by

(6.27) $\quad \Psi(\underset{\sim}{x}, t)=\int G\left(\underset{\sim}{x-x^{\prime}}, t\right) \Psi\left(\underset{\sim}{x^{\prime}}, 0\right) d^{3} x^{\prime}$,

which holds also if no approximation is made. In particular, to obtain the first cumulant-discard approximation for $\Psi(\underset{\sim}{x}, t)$, we substitute (6.20), with $k=0$, for $G(\underset{m}{x}, t)$ in $(6.27)$. This result holds for all $t$, since $\mathrm{B}_{0}=\infty$.

To illustrate our result, let us take

(6.28) $\quad \underset{\sim}{x}, 0)=\left(2 \pi d^{2}\right)^{-3 / 2} \exp \left[-\frac{1}{2}|\underset{\sim}{x}|^{2} / \alpha^{2}\right]$,

where $d$ is a measure of initial spread. In the first cumulant-discard approximation, we find

$$
\begin{aligned}
\underset{\sim}{x}, t)=-\left(2 \pi a^{2}\right)^{-3 / 2} \frac{a^{2}}{|\underset{m}{\mid x}|} \frac{\partial}{\partial|\underset{\sim}{x}|}\left\{\exp \left[-\frac{\left(|\underset{\sim}{x}|-v_{0} t\right)^{2}}{2 a^{2}}\right]\right. \\
\left.+\exp \left[-\frac{\left(||_{\sim}^{\mid x} \mid+v_{0} t\right)^{2}}{2 a^{2}}\right]\right\} .
\end{aligned}
$$

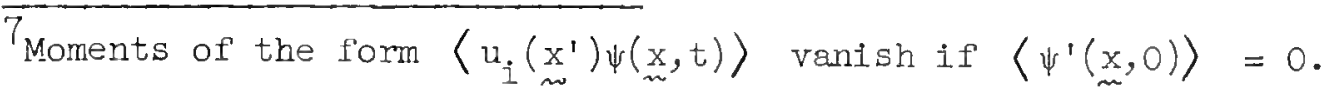


This predicts the devtopment of repative values for $\Psi(x, t)$. woreorer, we find from (6.29) that. while $/(x, t) a^{2} x$ is conserved is in the exact solution, $\int|\Psi(x, t)| a^{2} x$ srows indefinitely.

The closure (0.26) is ilentical wth that employed in a slitrty more general form by Reid $[22]$ to investitate the evolution of the wavenumber spectrum of an isotropically Astributed field $\psi^{\prime}(x, t)$ convected by isotropic turbulence. Re1d did not assume a static velocity fieid, as we have done, but insteal took a field obeying the inviscid Navierstokes equation. It is easily verified that our result (6.29) for the spread of an inhomogeneous scalar fleld is unchanged in this case. The introduction of viscostty does produce a change, but the unphysical behavior of $\Psi(\underset{\sim}{x}, t)$ persists. Our present results suggest that the cumulant-discard approximations for an isotropically distributed scalar field may lead to negative spectral densities for some wavenumbers. The explicit integration of Reid's spectral equation would shed light on this question.

6.4. Stochastic model approximations

The first stochastic model of the convection problem [16] has been investigated by Roberts [25]. The equation for $\mathrm{G}(\mathrm{x}, t)$, which we shall not derive here, is

$$
\begin{aligned}
& \text { (6.30) }\left(\frac{\partial}{\partial t}-k \nabla^{2}\right) G(x, t)=\int_{0}^{t} d s \int_{w}^{3} y U_{i j}(\underset{w}{x-y}) \frac{\partial G(x-y, t-s)}{\partial G(\underset{w}{y}, s)} \frac{\partial y_{j}}{j}, \\
& G(\underset{\sim}{x}, 0)=\delta^{3}(\underset{\sim}{x}) .
\end{aligned}
$$


It will be noticed that (6.30) resembles (6.18) but differs by being nonlinear in $G(\underset{m}{x}, t)$. For $t \ll \ell_{0} / v_{0}, U_{1, j}(\underset{m}{x-y})$ in $(6.30)$ may be replaced by $U_{1 j}(0)=\delta_{1 j} v_{0}^{2}$. Then the equation may be reduced by partial integration to the form

$$
\begin{aligned}
& \text { (6.3I) }\left(\frac{\partial}{\partial t}-k \nabla^{2}, G(\underset{m}{x}, t)=v_{0}^{2} \int_{0}^{t} d s \int d^{3} y G(\underset{m}{x}-y, t-s) \nabla^{2} G(\underset{\sim}{y}, s),\right. \\
& G(\underset{\sim}{x}, 0)=8^{3}(x),
\end{aligned}
$$

of which the solution is

(6.32) $\quad G(x, t)=\left(2 \pi v_{0} t\right)^{-2} \exp \left(k \nabla^{2} t\right)\left[\eta\left(||_{m} \mid / 2 v_{0} t\right)\left(4 v_{0}^{2} t^{2}-|x|_{m}^{2}\right)^{-I / 2}\right]$,

where

$$
\eta(s)=1 \quad(s<1), \quad \eta(s)=0 \quad(s \geqq 1) \text {. }
$$

Equation (6.32) represents a substantial improvement, over any of the iteration or cumulant-discard approximations we have examined, in that $G(\underset{m}{x}, t)$ is positive definite and is spread out over a region corresponding to the spread of the exact solution (6.5). In common with the previous approximations, the present one gives $\left\langle x_{1}{ }^{2}\right\rangle$ correctly in the range $t \ll \ell_{0} / v_{0}$ which we are considering. Apart from these virtues, however, the approximation is a poor one. Instead of falling off monotonically with the increase of $|x|, G \underset{m}{x}, t)$ is minimum at the origin, according to (6.32), and rises to a cusp and cut-off, at $|\mathrm{x}|=2 \mathrm{v}_{0} t$, whtch become infinitely sharp as $k \rightarrow 0$.

The behavior of the first stochastic model for long times is more satisfactory, and it represents a profound improvement over the previous 


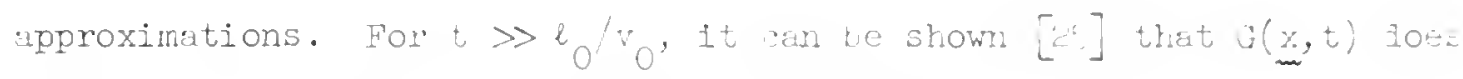
take the form $(6.7)$. The numiter at is elven by

$$
a=\left(2 v_{0}{ }^{l}\right)^{-1} \int_{0}^{\infty} d s \int a^{2} y U_{i i}(y)(j(y, s) .
$$

In order to evaluate this expression explicitly, we must integrate (6.30) to find $G(y, s)$ for all time ranges. The dominant contributions to (6.33) come from the region $\left(|y| \sim \ell_{0}, s \sim \ell_{0} / v_{0}\right)$. How accurate a quantitative approximation (6.33) represents is not known.

The behavior of $G(\underset{\sim}{x}, t)$ has been partially investigated for a tentative second stochastic model [16]. The approximation to the exact solution is very substantially improved for $t \ll \ell_{0} / v_{0}$. Outside of the asymptotic time ranges, the equations are very complicated.

\subsection{Formal relations among the approximations}

We stated in section 5 that the cumulant-discard approximations and the stochastic model approach both represented well-defined summations of selected terms from all orders of the iteration expansion. This is also true in the present problem, and the relations, in fact, take a simpler form. The iteration expansion of $G(\underset{m}{x}, t)$ consists of a sum of functionals of $\mathrm{U}_{i j}(\mathrm{x})$ of ascending degree. The contribution of $n t h$ degree arises as the average of a product of 2 n factors $u_{\sim}$. Therefore, according to the rules for evaluating moments of a normal distribution, this contribution consists of $(2 n) ! / 2^{n} n$ ! terms, one for each possible grouping of the $u_{m}$ factors into pairs. In the cumulant-discard and stochastic model approximations, some of these terms are discarded and others retained unaltered. 
We shall not state the selection rules here. 8 Instead, we have indicated in Table 1 the total number of terms of each order (degree in $U_{1 j}$ ) retained in each approximation. This is a particularly interesting measure for $t \ll \ell_{0} / v_{0}$ and $k=0$. Then all the $(2 n) ! / 2^{n} n !$ terms in the $n t h$ order of the iteration expansion give exactly equal contributions to $G(\underset{m}{x}, t)$.

\section{$\underline{T A B L E ~ I}$}

\section{Approximation}

Ist Cumulant-discard

and Cumulant-discard

3rd Cumulant-discard

Ist Stochastic Model
Terms retained of order $n$

$$
\begin{gathered}
1 \\
\frac{1}{2}\left[(3+\sqrt{6})^{n-1}+(3-\sqrt{6})^{n-1}\right] \\
(2 n) ! / n !(n+1) !
\end{gathered}
$$

It may be inferred from Table 1 that mere comprehensiveness of summation is not necessarily a good measure for the validity of an approximation. The third cumulant-discard approximation contains more terms for large $n$ than the first stochastic model. (The asymptotic numbers are of order $(3+\sqrt{6})^{\mathrm{n}}$ and $\mathrm{n}^{-3 / 2} \mathrm{~L}_{4}$, respectively.) However, it gives a poorer approximation at the long times where the high orders can contribute most strongly.

We should like to mention here that the structure of the iteration series for $G(x, t)$, and the infinite summations of terms from it which our approximations represent, have analogs in quantum field theory. In particular, the cumulant-discard approximations are close analogs to the Tamm-Dancoff approximations for the "one-nucleon propagator" in meson theory [26]. 


\section{CONCLUSION}

We have been concerned principally with a particular fom of the closure problem of turbulence theory: Given a simple initial distribution for the velocity field (a normal distribution), how can one obtain a simple analytical description of the distribution at later times? We have indicated that a simple exact description $1 \mathrm{~s}$ not known and is likely to be very difficult to find. This has led us to consider approximation approaches to the closure problem, and we have examined several in the context of isotropic turbulence.

The most straightforward approximation scheme we considered was based on expansion of the moments of the distribution in powers of the turbulent Reynolds number $R_{0}$ of the initial state. This scheme can be expected to yield good approximations only for the very restricted case $R_{0} \ll 1$. We have pointed out, however, that the Reynolds number, or iteration, expansion is of central theoretical interest at all $R_{0}$ as a formal standard of comparison for other approximations.

The second approach we examined was the expansion of the distribution in cumulants. It is more intimately and logically based on the statistical nature of the turbulence problem. The approximations arising from the cumulant expansion have not yet been exploited sufficiently to determine their validity. We have seen, however, that the consequences of these approximations in the problem of convection by a prescribed random velocity field do not provide a favorable prognosis for the1r validity in turbulence dynamics. The unfavorable results include negative probabilities and negative mean-square field-amplitude fluctuations. They suggest that the 
cumulants of the velocity distribution in turbulence dynamies may not decrease in importance with increasing order, when $R_{0}$ is large. It is very possible, however, that the convection example is misleading. Although it presents a closure problem similar to that of turbulence dynamics, there is the essential difference that the equations are linear in the dynamic variables. Whether nonlinearity in the dynamics serves to reduce or to accentuate the unphysical consequences of truncating the cumulant expansion remains to be determined.

If it turns out that the cumulant expansion does not provide satisfactory closure approximations for isotropic turbulence at high $\mathrm{R}_{\mathrm{O}}$, the outlook for finding acceptable alternative approximations of equal simplicity does not seem very favorable. This is illustrated by the third scheme we have considered, that of solving model problems which lead to exact closed moment equations. Negative probabilities and related troubles cannot arise in this scheme, but the price paid for the assurance is a quite substantial increase in the complexity of the final equations which must be solved.

In both the cumulant expansion scheme and the model scheme, the complexity of the approximations increases so rapidly with order that only the lowest approximations give much hope of practical utility. If they prove inadequate, the need for a genuinely fresh theoretical approach would seem strong. Perhaps there is an alternative representation of the statistical problem which leads to simpler and more natural approximations than those based on moments. Unfortunately, there seem to be only meager clues in the branches of statistical physics which have ylelded more graciously to analytical treatment. Turbulence at high Reynolds numbers 
is characterized by both strong nonlinearity and strone lissipation. This appears to be a particularly intractable combination of properties. We should like to close with the remark, made earlier, that isotropic turbulence, despite 1 ts geometrical simplicity, may constitute a particularly severe testing ground for closure approximations. As we have seen, the closure problem arises solely from the self-interaction of the fluctuating part of the total velocity field. In shear flow problems, the interaction of mean velocity fleld and fluctuating fleld makes a major contribution to the total dynamics. Perhaps this situation is one in which cruder closure approxtmations are permissible. None of the approximation schemes we have discussed is limited to homogeneous turbulence, and it is highly desirable that they be investigated for more general flows.

\section{ACKNOWLEDGMENT}

The author is very grateful to Dr. Edward Spiegel for many constructive suggestions. 


\section{REFERENCES}

1. G.K. Batchelor, "Diffusion in a field of homogeneous turbulence. I. Eulerian analysis", Australian J. Sci. Res. 2, 437 (1949).

2. _._._. "Diffusion in a field of homogeneous turbulence. II. The relative motion of particles", Proc. Camb. Phil. Soc. 48, 345 (1952).

3. _. "The effect of homogeneous turbulence on material lines and surfaces", Proc. Roy. Soc. London, A 213, 349 (1952).

4. _._. "The Theory of Homogeneous Turbulence", Cambridge, University Press, 1953.

5. R. Betchov, "An inequality concerning the production of vorticity in isotropic turbulence", J. Fluid Mech. 1, 497 (1956).

6. S. Chandrasekhar, "A theory of turbulence", Proc. Roy. Soc. London, A 229, 1 (1955).

7. R.G. Deissler, "On the decay of homogeneous turbulence before the ff.nal period", Phys. Fluids. 2, 111 (1958).

8. _. "A theory of decaying homogeneous turbulence", Phys. Fluids 2, 176 (1960).

a. W. Leisenberg, "Zur statistischen Theory der Turbulenz", Z. Physik, 124,628 (1948).

10. E. Hopf, "Statistical hydromechanics and functional calculus", J. Rat. Mech. Anal. I, 87 (1952).

11. _L_Proceedings of Symposium on Hydrodynamic Instability", edited by G. Birkhoff, Providence, American Mathematical Society, to be published.

12. A.N. Kolmogorov, "The local structure of turbulence in an incompressible viscous fluid for very large Reynolds numbers", C.R. Acad. Sci. U.S.S.R. 30, 301 (1941).

13. , "Dissipation of energy in locally isotropic turbulence", C.R. Acad. Sci.U.S.S.R. 32, 16 (1941). 
14. R. Krajchnan, "Relation of fourth-order to second-order moments in stationary isotropic turbulence", Phys. Rev. 107, 1485.(1957).

15. _._. "The structure of isotropic turbulence at very high Reynolds numbers", J. Flu1d Mech. 2, 497 (1959). [See also:

"Second Symposium on Naval Hydrodynamics", edited by R. Cooper, Publication ACR-38, Office of Naval Research-Department of the Navy, Washington, D.C., 1958.]

16. _Lynamics of nonlinear stochastic systems", J. Math. Phys. 2, 124 (1961).

17. J. Laufer, "On the structure of fully developed turbulent pipe flow", Report 1174, Nat. Adv1s. Comm. Aero., 1954.

18. C.C. Lin, "The Theory of Hydrodynamic Stability", Cambridge, University Press, 1955.

19. W.V.R. Malkus, "Outline of a theory of turbulent shear flow", J. Fluid Mech. I, 52i (1956).

20. M. Millionstchikov, "On the theory of homogeneous isotropic turbuIence", C.R. Acad Sci. U.S.S.R. 32, 615 (1941).

21. I. Proudman and W.H. Reid, "On the decay of a normally distributed homogeneous turbulent velocity field", Phil. Trans. Roy. Soc. London, A $247,163(1954)$.

22. W.H. Reid, "On the stretching of material lines and surfaces in isotropic turbulence with zero fourth cumulants", Proc. Camb. Phil. Soc., 51, $350(1955)$.

23. 0. Reynolds, "On the dynamical theory of incompresstble viscous fluids and the determination of the criterion", Phil. Trans. Roy. Soc. London, A I86, 123 (1894).

24. P.H. Roberts, "On the application of a statistical approximation to the theory of turbulent diffusion", J. Rat. Mech. Anal. 6, 781 (1957). 25. , "Analytical theory of turbulent diffusion", to appear. [Issued also as Report HSN-2, New York University, Institute of Mathematical Sciences, Division of Electromagnetic Research, June 1960.] 
26. S. Schweber, H. Bethe, and F. de Hoffmann, "Mesons and Fields, Vol. I. Fields", New York, Row, Peterson, 1955.

27. T. Tatsumi, "The theory of decay process of incompressible isotropic turbulence", Proc. Roy. Soc. London, A 239, 16 (1957).

28. , "Energy spectra in magneto-fluid dynamic turbulence", Rev. Mod. Phys. 32, 807 (1960).

29. G.I. Taylor, "Diffusion by continuous movements", Proc. Lond. Math. Soc., series 2, 20, 196 (1922).

30 . , "Statistical theory of turbulence, Parts 1-4", Proc. Roy. Soc. London, A 151, 421 (1935).

31. R.C. Tolman, "The Principles of Statistical Mechanics", New York, Oxford University Press, 1938.

32. A.A. Townsend, "The Structure of Turbulent Shear Flow", Cambridge, University Press, 1956.

Arí 1 \% 1961

Date Due

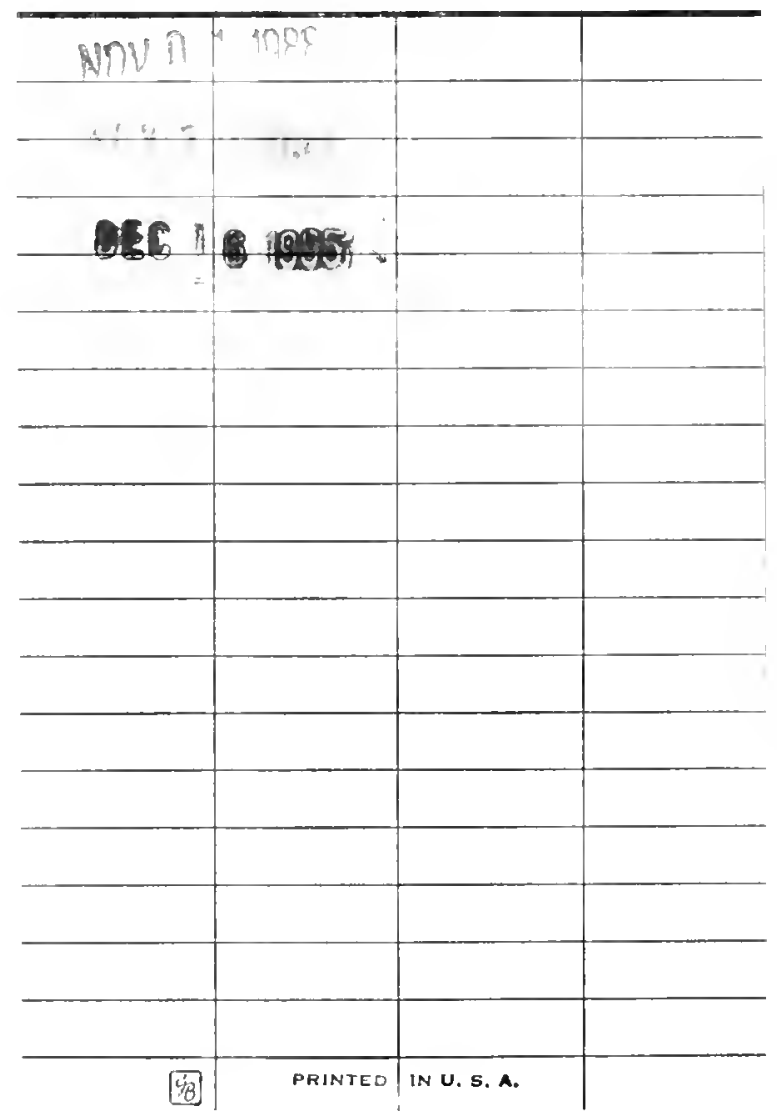


ISN - 3

Kraichnan

The closure problem of turbulence theory

NIT

ISIT-3

Iraichan

AUTHOR

Tho closure problem of

TICLE

turbulence theory

DÁt (II)

$l^{\prime \prime}$

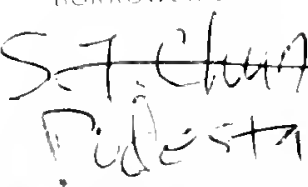

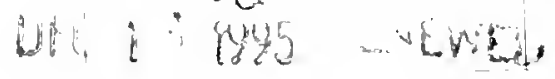

N. Y. U. Institute of Mathematical Sciences

25 Waverly Place New York 3, N. Y. 

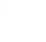

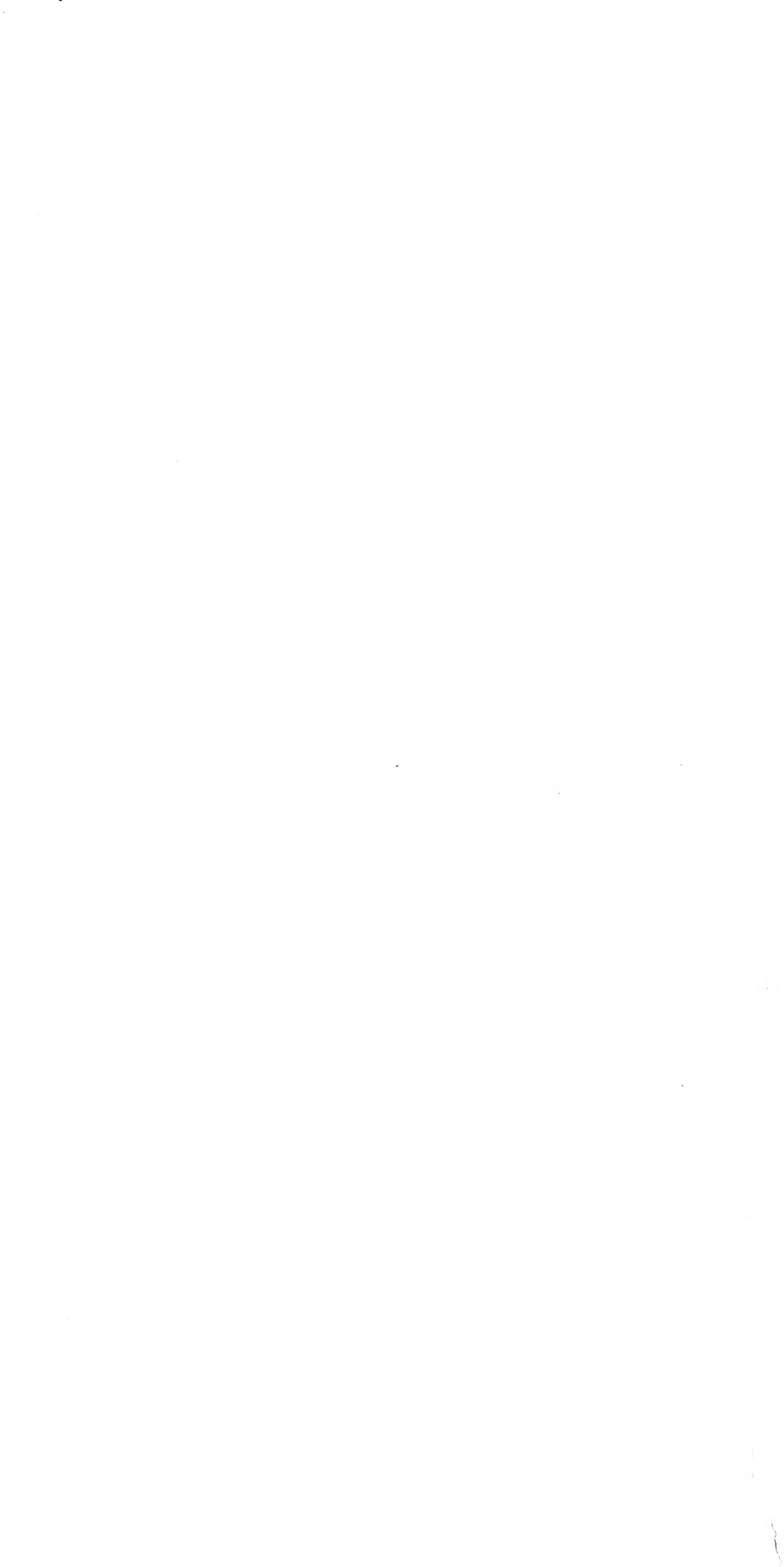

